\title{
Alcohol Use among Chinese Men Who Have Sex with Men: An Epidemiological Survey and Meta-Analysis
}

\author{
Yu Liu, ${ }^{1}$ Han-Zhu Qian, ${ }^{1,2}$ Yuhua Ruan, ${ }^{3}$ Lu Yin, ${ }^{1}$ Juntao Ma, ${ }^{4}$ Kapil Dahiya, \\ Wensheng Fan, ${ }^{5}$ Yiming Shao, ${ }^{3}$ and Sten H. Vermund ${ }^{1,6}$ \\ ${ }^{1}$ Vanderbilt Institute for Global Health, Vanderbilt University, Nashville, TN 37203, USA \\ ${ }^{2}$ Division of Epidemiology, Department of Medicine, Vanderbilt University School of Medicine, Nashville, TN 37203, USA \\ ${ }^{3}$ State Key Laboratory for Infectious Disease Prevention and Control, National Center for AIDS/STD Control and Prevention, \\ Chinese Center for Disease Control and Prevention, Collaborative Innovation Center for Diagnosis and \\ Treatment of Infectious Diseases, Beijing 102206, China \\ ${ }^{4}$ Medical Library of Chinese People's Liberation Army, Beijing 100039, China \\ ${ }^{5}$ Department of Public Health, College of Health and Human Services, Western Kentucky University, Bowling Green, KY 42101, USA \\ ${ }^{6}$ Department of Pediatrics, Vanderbilt University School of Medicine, Nashville, TN 37203, USA
}

Correspondence should be addressed to Sten H. Vermund; sten.vermund@vanderbilt.edu

Received 18 October 2013; Accepted 12 January 2014; Published 11 March 2014

Academic Editor: Yujiang Jia

Copyright (c) $2014 \mathrm{Yu}$ Liu et al. This is an open access article distributed under the Creative Commons Attribution License, which permits unrestricted use, distribution, and reproduction in any medium, provided the original work is properly cited.

\begin{abstract}
The HIV/AIDS epidemic among Chinese men who have sex with men (MSM) has become a significant public health concern. Knowledge of alcohol consumption in this population is limited. In this study, 1,155 Chinese MSM were surveyed to assess alcohol use and its correlates. A meta-analysis was also performed to aggregate pooled prevalence of current alcohol use. MSM who were unmarried (aOR: 1.87; 95\% CI: 1.29-2.71) or unemployed/retired (aOR: 2.77; 95\% CI: 1.73-4.45) were more likely to drink alcohol more than once per week. MSM who consumed alcohol more than once per week were more likely to use $\operatorname{drug}(P<0.01)$, have sex with women $(P<0.01)$, have unprotected insertive $(P=0.04)$ or receptive $(P=0.03)$ anal sex with men, have more than 10 lifetime male sex partners $(P<0.01)$, predominantly practice insertive anal sex $(P<0.01)$, and trade sex for money $(P<0.01)$. Pooled overall alcohol use prevalence was $32 \%$. Pooled prevalence for MSM who drank alcohol more than once per week and who drank alcohol before sex with male partners was $23 \%$. Our findings provide the basis for further exploring the alcohol-HIV association and developing risk reduction interventions.
\end{abstract}

\section{Introduction}

HIV transmission is shifting in China from a preponderance of persons who inject drugs to those persons who acquire HIV sexually; homosexual transmission among men who have sex with men (MSM) is of particular concern [1]. The proportion of new HIV cases among Chinese MSM has surged from $0.2 \%$ in 2001 to $29.4 \%$ in 2011 , and the major risk in this population is unprotected anal sex [2-4]. Prospective cohort studies have shown that Chinese MSM who experienced unprotected anal intercourse or had multiple male partners were 3-10 times more likely to encounter HIV seroconversion than MSM not practicing these risk behaviors [5-8].

Alcohol abuse is a factor in a variety of medical, social, and public health problems. Global evidence suggests that alcohol use is common among MSM, and can be associated with high risk behaviors for HIV and for other sexually transmitted infections (STI), such as unprotected sex, commercial sex, sexual violence, and sex with multiple concurrent partners [9-15].

Due to traditional social norms and booming economic development, alcohol is used commonly among Chinese men, especially those living in rural areas $(26.4 \%$ among 
rural men versus $12.6 \%$ among urban men) [16]. Data from the China Chronic Disease and Risk Factor Surveillance study suggests that the prevalence of current drinking was $35.7 \%$, higher among males (55.6\%) than females $(15.0 \%)$ [17]. Knowledge about prevalence of alcohol consumption prevalence and its important correlates among Chinese MSM is limited [18-20]. In this study, we sought to (1) evaluate the association of demographic factors with alcohol use among Chinese MSM; (2) compare risky behaviors among MSM who drank and who did not drink alcohol; and (3) perform a metaanalytic literature review to better estimate the prevalence of alcohol consumption among Chinese MSM with available published studies.

\section{Materials and Methods}

2.1. Study Design and Population. Our cross-sectional study was conducted among MSM in Beijing, China, during 2010 and 2011. The study design and study population were described in detail elsewhere [37]. In brief, the study participants were recruited from the community through a local gay-oriented community-based organization (CBO) and through referrals from ongoing epidemiological study. The primary objective of the study was to assess the relationship between male circumcision and HIV/HPV risks and a secondary objective was to explore alcohol use among MSM. Each study participant received genital exam on circumcision status, testing of blood and anal and genital swab specimens, and a questionnaire interview. Written informed consent was obtained. This study was approved by the institutional review boards of the National Center for AIDS/STD Control and Prevention of the Chinese Center for Disease Control and Prevention and Vanderbilt University School of Medicine.

2.2. Data Collection. A questionnaire interview collected data on sociodemographic characteristics (e.g., age, ethnicity, marital status, occupation, education, Beijing residence, and duration of living in Beijing), current alcohol drinking status (at least once per week), HIV risk behaviors (e.g., illicit drug use, sexual orientation, number of male or female sex partners and concurrent partners, specific sexual activities and condom use, anal sex role with male partners, commercial sex, and forced sex), and history of STI. Laboratory testing of HIV and syphilis was conducted according to Chinese national protocol.

For the meta-analysis on prevalence of current alcohol use among MSM in China, we searched both English and Chinese publications up to September 15, 2013, mainly in two databases: MEDLINE via PubMed and Wanfang Data, and we also searched Google Scholar for additional publications. The following search strategy was used to identify articles: ("China" OR "Chinese") AND ("MSM" OR "men who have sex with men" OR "gay" OR "homosexual”) AND ("ethanol" OR "alcohol" OR "alcohols"). We also conduct reviews on the reference list of all eligible articles to identify additional literature relevant to the topic. Articles were considered eligible for inclusion if they were original epidemiologic studies conducted among MSM in China with reported alcohol use prevalence and/or had crucial statistics permitting us to compute prevalence. Two reviewers (Yu Liu and Han-Zhu Qian) examined relevant abstracts and determined whether they met criteria for inclusion.

2.3. Statistical Analysis. We sought to identify sociodemographic factors associated with alcohol consumption among Chinese MSM. First, we defined alcohol drinkers as someone who drank alcohol at least once per week in the past 4 weeks, and we compared sociodemographic factors between alcohol drinking MSM and nondrinkers in univariate logistic regression analysis. Significant factors were further evaluated by being fitting into multivariable logistic regression model. In each multivariable logistic model, potential confounders were adjusted a priori using direct acyclic graph (DAG). Odds ratios (OR) and 95\% confidence intervals (CI) were calculated for both univariate and multivariable logistic regressions. We also compared risky behaviors between alcohol drinkers and nondrinkers by using Chi-squared test or a two-tailed Fisher's exact test.

In meta-analysis, we used three definitions on alcohol consumption: (1) the loose definition on drinking or not drinking alcohol which could mean different frequency and volume in the original papers; (2) ever drinking alcohol before sex; (3) drinking alcohol for at least once a week, which is consistent with that in our epidemiological survey. To account for the potential heterogeneity of studied population in each study reflected by different geographic locations, ethnicity, age group, and so forth, a random-effect model using the DerSimonian and Laird method [38, 39] was used to aggregate effect sizes to estimate the overall pooled prevalence and corresponding 95\% CIs. Two separate random-effect models were also used to summarize the prevalence of alcohol consumption before sex with male sexual partners and alcohol use at least once per week. To quantify the heterogeneity of effect sizes over all included studies, $I^{2}$ statistics were calculated. Funnel plots were used to graphically examine signal of potential publication bias. Egger tests were performed to test the funnel plot asymmetry.

STATA 12.0 (StataCorp LP, College Station, Texas) was used for all statistical analyses.

\section{Results}

3.1. Demographics and Alcohol Use among MSM in Beijing. A total of 1,155 MSM provided informed consent to participate in the original study. Fifteen participants had missing information on alcohol drinking; therefore, 1140 (98.7\%) participants were included in the analysis. Six percent were non-Han minority ethnics; $27 \%$ were married; $53 \%$ had ever attended college; $66 \%$ had Beijing residency; $59 \%$ had lived in Beijing for more than 4 years. Age ranged from 18 to 68 years ( median $=30$ years $)$. The prevalence of drinking alcohol at least once a week was $23 \%$.

3.2. Sociodemographic Factors Associated with Alcohol Consumption. Univariate analyses suggested the following factors to be associated with alcohol consumption: age, marriage, education, occupation, and sexual orientation. After controlling for age, education, and Beijing residency, being 
TABLE 1: Sociodemographic predictors for alcohol drinking among men who have sex with men in Beijing, China $(N=1140)$.

\begin{tabular}{|c|c|c|c|c|}
\hline \multirow{2}{*}{ Sociodemographic factors ${ }^{\mathrm{a}}$} & \multicolumn{2}{|c|}{ Alcohol consumption $(N, \%)$} & \multirow{2}{*}{ Crude OR (95\% CI) } & \multirow{2}{*}{ Adjusted OR $(95 \% \mathrm{CI})^{\mathrm{b}}$} \\
\hline & $\begin{array}{c}<\text { once per week } \\
(N=875)\end{array}$ & $\begin{array}{c}\geq \text { once per week } \\
\quad(N=265)\end{array}$ & & \\
\hline Age (year) & & & $1.03(1.01,1.05)$ & $1.00(0.98,1.02)$ \\
\hline Median, IQR & $30(26-35)$ & $32(27-39)$ & & \\
\hline \multicolumn{5}{|l|}{ Ethnicity } \\
\hline Han Chinese & $821(93.8)$ & $252(95.1)$ & Reference & \multirow{2}{*}{ - } \\
\hline Non-Han & $54(6.2)$ & $13(4.9)$ & $0.78(0.42,1.46)$ & \\
\hline \multicolumn{5}{|l|}{ Marital status } \\
\hline Currently married & $205(23.4)$ & $105(39.6)$ & Reference & Reference \\
\hline Currently unmarried & $670(76.6)$ & $160(60.4)$ & $2.14(1.60,2.87)$ & $1.87(1.29,2.71)$ \\
\hline \multicolumn{5}{|l|}{ Education (years of schooling) } \\
\hline College and above $(>12)$ & $480(54.9)$ & $122(46.0)$ & Reference & \\
\hline Senior high school (10-12) & $240(27.5)$ & $85(32.1)$ & $1.39(1.01,1.91)$ & $1.24(0.90,1.73)$ \\
\hline Junior high school (7-9) & $133(15.2)$ & $48(18.1)$ & $1.42(0.97,2.09)$ & $1.21(0.81,1.80)$ \\
\hline Primary school $(\leq 6)$ & $21(2.4)$ & $10(3.8)$ & $1.87(0.86,4.08)$ & $1.43(0.64,3.21)$ \\
\hline \multicolumn{5}{|l|}{ Occupation } \\
\hline Employed & $752(86.0)$ & $204(77.0)$ & Reference & Reference \\
\hline Unemployed/retired & $47(5.3)$ & $34(13.2)$ & $2.78(1.76,4.42)$ & $2.77(1.73,4.45)$ \\
\hline Student & $44(5.1)$ & $4(1.5)$ & $0.86(0.44,1.65)$ & $0.84(0.42,1.77)$ \\
\hline Other & $32(3.6)$ & $22(8.3)$ & $1.52(0.86,2.73)$ & $1.53(0.90,2.88)$ \\
\hline \multicolumn{5}{|l|}{ Beijing residency } \\
\hline No & $292(33.4)$ & $94(35.6)$ & Reference & \multirow{2}{*}{-} \\
\hline Yes & $583(66.6)$ & $170(64.4)$ & $0.91(0.68,1.21)$ & \\
\hline \multicolumn{5}{|c|}{ Duration of living in Beijing (years) } \\
\hline$\leq 4$ & $365(41.7)$ & $100(37.7)$ & Reference & \multirow[t]{2}{*}{-} \\
\hline$>4$ & $510(58.3)$ & $165(62.3)$ & $1.18(0.89,1.57)$ & \\
\hline \multicolumn{5}{|l|}{ Sexual orientation } \\
\hline Homosexual & $602(69.8)$ & $156(59.3)$ & Reference & \\
\hline Heterosexual & $9(1.0)$ & $4(1.5)$ & $1.71(0.52,5.64)$ & $1.60(0.48,5.33)$ \\
\hline Bisexual & $252(29.2)$ & $103(39.2)$ & $1.58(1.18,2.11)$ & $1.28(0.93,1.75)$ \\
\hline
\end{tabular}

IQR: interquartile range; OR: odds ratio; CI: confidence interval.

${ }^{\mathrm{a}}$ Sample size may vary due to missing data. ${ }^{\mathrm{b}}$ Adjusted for age, education, and Beijing residency.

currently unmarried (adjusted OR [aOR]: 1.87; 95\% CI: $1.29-$ 2.71) and being unemployed/retired (aOR: 2.77 ; $95 \%$ CI: $1.73-$ 4.45) were significantly associated with drinking alcohol at least once per week (Table 1).

3.3. Comparison of HIV Risk Behaviors between MSM Who Drank and Did Not Drink Alcohol. Alcohol drinking MSM were more likely to use illicit drug use $(6.0 \%$ versus $1.9 \%$; $P<0.01)$, to have female sexual partners in the past 6 months (61.5\% versus $40.7 \% ; P<0.01)$, to have unprotected insertive $(23.2 \%$ versus $17.6 \% ; P=0.04)$ or receptive $(26.8 \%$ versus $18.3 \%$; $P=0.03$ ) anal sex with men in the past 6 months, to have $\geq 10$ lifetime male sex partners $(66.8 \%$ versus $59.8 \% ; P=0.04)$, to experience insertive anal sex with male partner in the past 6 months $(80.4 \%$ versus $63.7 \% ; P<$ $0.01)$, to predominantly practice insertive anal sex $(52.7 \%$ versus $41.0 \%$; $P<0.01$ ), and to trade sex for money in the past 12 months $(10.2 \%$ versus $3.5 \% ; P<0.01)$ MSM who drank alcohol were less likely to have more than one lifetime female sex partner $(70.2 \%$ versus $76.2 \% ; P=0.04)$ and to be HIV (19.8\% versus $27.3 \%$; $P=0.02)$ infected or syphilis seropositive (21.2\% versus $27.7 \% ; P=0.04$ ) (Table 2 ).

3.4. Meta-Analysis of Literature on Alcohol Consumption among Chinese MSM. Our search strategy identified 2527 studies; 19 met the inclusion criteria and were included for computing the meta-analytic outcome of alcohol use prevalence (Table 3). All these 19 studies used cross-sectional design, and current alcohol use rates ranged from $16.5 \%$ to $79.7 \%$; the pooled prevalence was $32 \%$ (effect size: $0.32 ; 95 \%$ CI: 0.25-0.40) (Figure 1). Five studies measured only general alcohol use status (yes versus no) [21, 24, 26, 31, 34]. Seven studies measured ever use of alcohol before sex [22, 23, 32, 35, 36 ], and the pooled prevalence was $23 \%$ (effect size: $0.23 ; 95 \%$ 
TABLE 2: Comparison of HIV risky behaviors between alcohol drinking and nondrinking men who have sex with men in Beijing, China $(N=1140)$.

\begin{tabular}{|c|c|c|c|}
\hline \multirow{2}{*}{ Risk factors ${ }^{\mathrm{a}}$} & \multicolumn{2}{|c|}{ Alcohol consumption $(N, \%)$} & \multirow{2}{*}{$P$ value } \\
\hline & <once per week & $\geq$ once per week & \\
\hline Illicit drug use & & & $<0.01$ \\
\hline No & $856(98.1)$ & $249(94.0)$ & \\
\hline Yes & $17(1.9)$ & $16(6.0)$ & \\
\hline Sexual orientation & & & $<0.01$ \\
\hline Homosexual & $602(69.8)$ & $156(59.3)$ & \\
\hline Heterosexual & $9(1.0)$ & $4(1.5)$ & \\
\hline Bisexual & $252(29.2)$ & $103(39.2)$ & \\
\hline Sex with female partner in past 6 months & & & $<0.01$ \\
\hline No & $519(59.3)$ & $102(38.5)$ & \\
\hline Yes & $356(40.7)$ & $163(61.5)$ & \\
\hline Condom use with female partner in past 6 months & & & 0.67 \\
\hline Every time & $56(27.5)$ & $31(30.4)$ & \\
\hline Very often & $20(9.8)$ & $6(5.9)$ & \\
\hline Rarely & $29(14.2)$ & $16(15.7)$ & \\
\hline Never & $99(48.5)$ & $49(48)$ & \\
\hline Number of lifetime female sex partners & & & 0.04 \\
\hline$<2$ & $208(23.8)$ & $79(29.8)$ & \\
\hline$\geq 2$ & $667(76.2)$ & $186(70.2)$ & \\
\hline Oral sex with male partner in past 6 months & & & 0.82 \\
\hline No & $147(16.8)$ & $43(16.2)$ & \\
\hline Yes & $726(83.2)$ & $222(83.8)$ & \\
\hline Condom use during oral sex with male partner in past 6 months & & & 0.49 \\
\hline Every time & $69(9.5)$ & $167(75.2)$ & \\
\hline Very often & $36(5.0)$ & $28(12.6)$ & \\
\hline Rarely & $72(10.0)$ & $7(3.2)$ & \\
\hline Never & $546(75.5)$ & $20(9.0)$ & \\
\hline Insertive anal sex with male partner in past 6 months & & & $<0.01$ \\
\hline No & $313(36.3)$ & $52(19.6)$ & \\
\hline Yes & $549(63.7)$ & $213(80.4)$ & \\
\hline Condom use during insertive anal sex with male partner in past 6 months & & & 0.04 \\
\hline Every time & $324(59.5)$ & $117(55.5)$ & \\
\hline Very often & $125(22.9)$ & $45(21.3)$ & \\
\hline Rarely & $54(9.9)$ & $36(17.0)$ & \\
\hline Never & $42(7.7)$ & $13(6.2)$ & \\
\hline Receptive anal sex with male partner in past 6 months & & & 0.94 \\
\hline No & $376(43.6)$ & $114(43.3)$ & \\
\hline Yes & $486(56.4)$ & $149(56.7)$ & \\
\hline Condom use during receptive anal sex with male partner in past 6 months & & & 0.03 \\
\hline Every time & $296(60.8)$ & $74(49.7)$ & \\
\hline Very often & $102(20.9)$ & $35(23.5)$ & \\
\hline Rarely & $44(9.1)$ & $25(16.8)$ & \\
\hline Never & $45(9.2)$ & $15(10.0)$ & \\
\hline Number of lifetime male sex partners & & & $<0.01$ \\
\hline$<10$ & $352(40.2)$ & $88(33.2)$ & \\
\hline$\geq 10$ & $523(59.8)$ & $177(66.8)$ & \\
\hline Anal sexual role with male partner & & & $<0.01$ \\
\hline Mainly/definitely receptive & $299(35.4)$ & $59(23.1)$ & \\
\hline Mainly/definitely insertive & $346(41.0)$ & $135(52.7)$ & \\
\hline
\end{tabular}


TABLE 2: Continued.

\begin{tabular}{|c|c|c|c|}
\hline \multirow{2}{*}{ Risk factors $^{\mathrm{a}}$} & \multicolumn{2}{|c|}{ Alcohol consumption $(N, \%)$} & \multirow{2}{*}{$P$ value } \\
\hline & $<$ once per week & $\geq$ once per week & \\
\hline Versatile $^{\mathrm{b}}$ & $199(23.6)$ & $62(24.2)$ & \\
\hline Multiple concurrent male partners in past 12 months & & & 0.27 \\
\hline No & $807(93.0)$ & $241(90.9)$ & \\
\hline Yes & $61(7.0)$ & $24(9.1)$ & \\
\hline Traded sex for money in the past 12 months & & & $<0.01$ \\
\hline No & $839(96.5)$ & $238(89.8)$ & \\
\hline Yes & $30(3.5)$ & $27(10.2)$ & \\
\hline Forced sex with any male partner & & & 0.08 \\
\hline No & $843(97.0)$ & $251(94.7)$ & \\
\hline Yes & $26(3.0)$ & $14(5.3)$ & \\
\hline Self-reported history of sexually transmitted diseases & & & 0.84 \\
\hline No & $588(69.7)$ & $180(70.3)$ & \\
\hline Yes & $256(30.3)$ & $76(29.7)$ & \\
\hline Syphilis seropositive & & & 0.04 \\
\hline No & $633(72.3)$ & $208(78.8)$ & \\
\hline Yes & $242(27.7)$ & $56(21.2)$ & \\
\hline HIV seropositive & & & 0.02 \\
\hline No & $635(72.7)$ & $211(80.2)$ & \\
\hline Yes & $238(27.3)$ & $52(19.8)$ & \\
\hline
\end{tabular}

${ }^{a}$ Sample size varies due to missing data.

${ }^{\mathrm{b}}$ Versatile indicates mixed receptive and insertive anal sex roles.

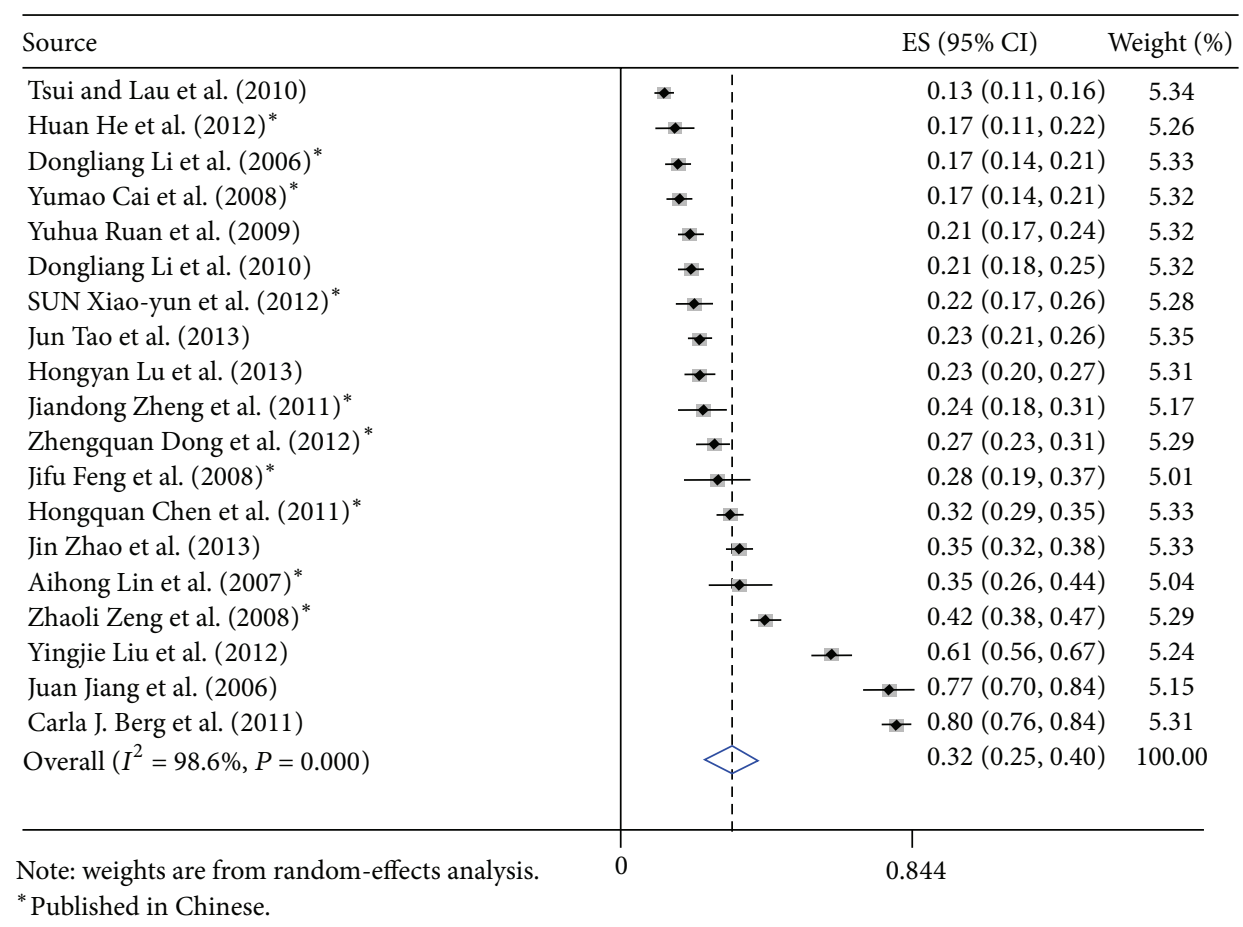

FIGURE 1: Forest plots of studies reporting prevalence of current alcohol consumption among Chinese men who have sex with men. 


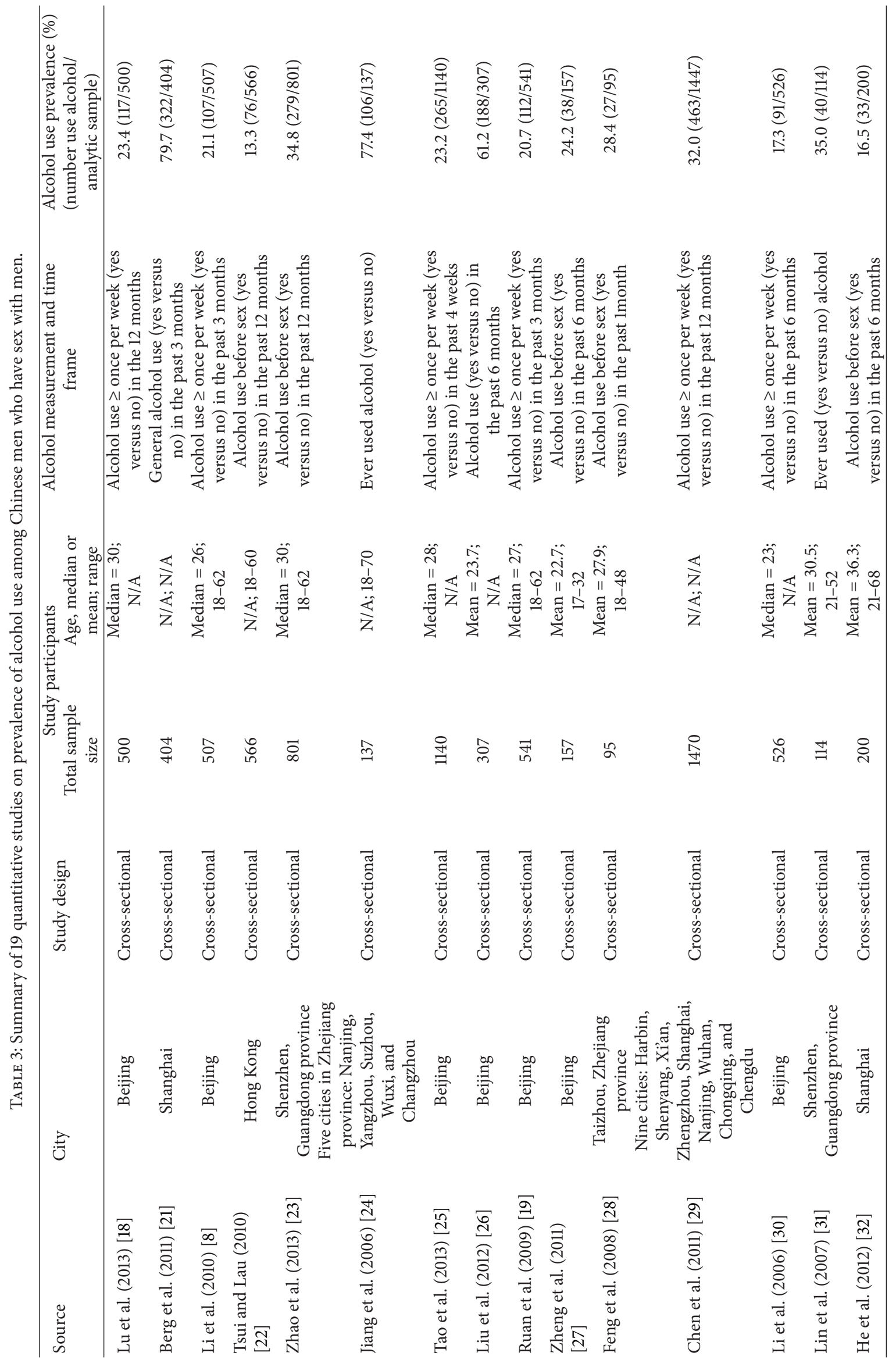




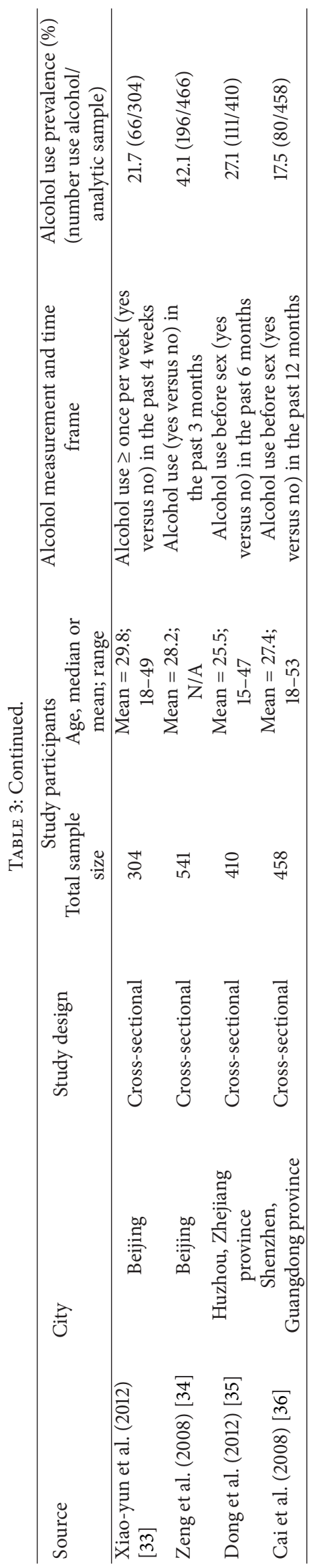




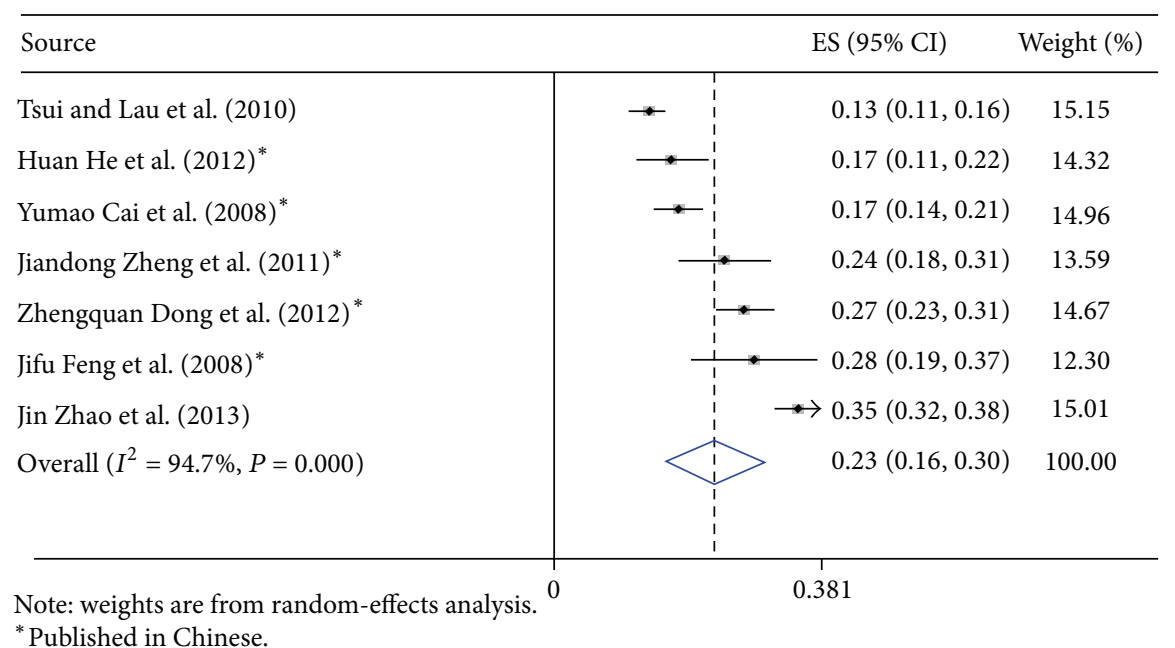

FIGURE 2: Forest plot of studies reporting prevalence of alcohol consumption before sex with male partners among Chinese men who have sex with men.

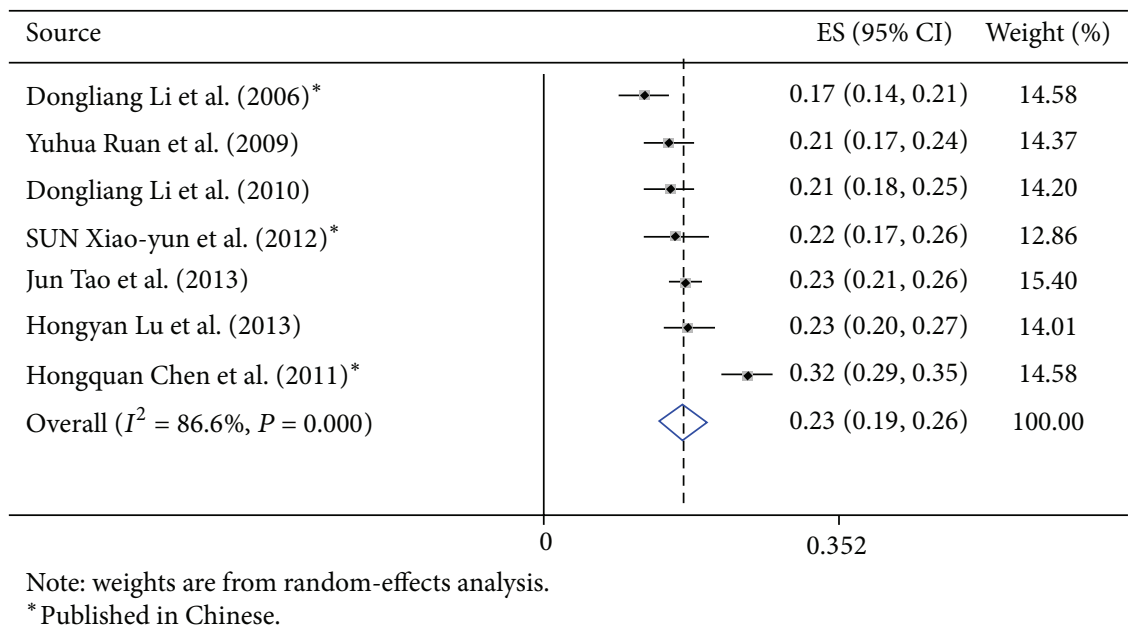

FIGURE 3: Forest plot of studies reporting prevalence of alcohol consumption $\geq$ once a week among Chinese men who have sex with men.

CI: 0.19-0.26) (Figure 2). Seven studies measured alcohol use at least once per week $[8,18,19,25,30,33]$, and the pooled prevalence was also 23\% (effect size, 0.23 ; 95\% CI, 0.19-0.26) (Figure 3). Large heterogeneity was observed among these studies $\left(I^{2}=98.6 \%, P<0.01\right)$. The funnel plot does not suggest significant publication bias (Egger's test $P$ value $=$ $0.13)$.

\section{Discussion}

In our survey of Chinese MSM living in Beijing, 23\% of participants reported alcohol consumption at least once a week, and no association was noted with HIV or syphilis seropositivity. Unmarried and unemployed MSM were more likely to drink alcohol. Alcohol drinking MSM were more likely to report risky behaviors including illicit drug use, larger number of sexual partners, and unprotected sex. Alcohol has been consumed worldwide for a variety of recreational and psychological reasons. Because of its psychogenic nature and capability of impairing decision making [40], alcohol use has been associated with HIV/STI risk behaviors in both developed and developing countries [13, 14]. MSM may use alcohol for sexual arousal and expectation of casual sex [41]. Our findings are consistent with other studies that a higher frequency of alcohol consumption was associated with a greater likelihood of engaging in unprotected anal intercourse $[2,18,19,42,43]$. No association was seen with HIV or syphilis infections per se, suggesting transmission dynamics to be more complex that could be unraveled here.

Understanding of Chinese culture is particularly important in researching alcohol use among MSM [21]. Homosexual relationships are not legal in China and homosexuality is stigmatized in the general public because of traditional taboos and cultural discrimination [44]. Hence, Chinese MSM typically endure social isolation, stress, and low self-esteem; they suffer from psychological syndemics, as reported elsewhere in the world, representing multiple psychological vulnerabilities and related adverse health outcomes $[9,45]$. 
With under one-quarter of Beijing MSM imbibing alcohol at least weekly, our survey found a lower alcohol consumption prevalence than the pooled prevalence among Chinese MSM that we calculated in our literature meta-analysis (32\%; 95\% CI: 25-40\%). However, our findings are consistent with the geographic differences noted for alcohol consumption in the general Chinese population, since men in large cities are less likely to drink alcohol than in rural regions [16].

Our meta-analysis demonstrated that alcohol is often drunk before sex among Chinese MSM (pooled prevalence 23\%), a well-known global risk factor for HIV and STIs. A longitudinal study in Rakai, Uganda, found the risk of contracting HIV among participant who used alcohol before sex was increased by $50 \%$ [46]. In China, about $30 \%$ of men who sought sex from female sex workers reported alcohol drinking before sex, and alcohol use was associated with less condom use and increased HIV/STIs in that context [47]. Since knowledge on factors associated with alcohol use before sex with male partners is scarce among Chinese MSM [48], more work is needed, particularly to assess casual alcohol use from heavy use, since it is the latter that is most commonly associated with high HIV/STI risks $[49,50]$. Whether community-based health education may be helpful in raising consciousness about alcohol and higher risk taking among MSM is not known $[51,52]$.

There are several limitations in our study. First, alcohol consumption was based on self-reporting, and the data may be subjected to recall bias; since our study focused on circumcision, the alcohol use questions were not comprehensivewe did not measure the type and amount of alcohol that MSM consumed in the study [13,53-58]. Second, the crosssectional study design did not allow assessing the temporal relationship between alcohol consumption and HIV risk behaviors. Third, the study participants in the epidemiological survey were a convenience sample, including mixed sample from the community (HIV-unknown MSM) and from ongoing study (HIV-infected MSM), so no inference about true drinking prevalence can be justified, but the analysis on the risk factors for alcohol use provided useful information for developing intervention programs. Fourth, the studies included in the meta-analysis for computing pooled prevalence on alcohol drinking at least once per week were mostly conducted in Beijing; thus, the sample representativeness may limit the generalizability of our finding. Finally, there is high heterogeneity on alcohol use among included studies; it could be from within-study variance due to population characteristics and from between-study variances due to geographic variation and different metrics and mechanism of ascertaining alcohol use. However limited information is present to explore these factors and too few findings to warrant further stratified analyses. Despite these limitations, our study presents one of few large studies of investigating alcohol use and its correlates among MSM in China and ours is the first to assess alcohol use prevalence among Chinese MSM with a meta-analysis. Therefore, our study provides useful information for HIV prevention programs targeting this population and can signpost future work and intervention.
In summary, HIV and STI rates are rising among Chinese MSM and alcohol consumption is associated with increased risk-taking behaviors, likely through sexual disinhibition. To better understand the impact of alcohol consumption on sexual behaviors and HIV transmission among MSM, future studies should employ a prospective research design and more refined measures of alcohol use.

\section{Conflict of Interests}

The authors declare that there is no conflict of interests regarding the publication of this paper.

\section{Acknowledgments}

This study was sponsored by the National Natural Science Foundation of China (81273188), the Vanderbilt Clinical \& Translational Research Scholars (VCTRS) Program (UL1 TR000445 and KL2TR000446), Grants from U.S. NIH (R01AI09462) and the Ministry of Science and Technology of China (2012ZX10004-904 and 2012ZX10001-002), and Chinese State Key Laboratory for Infectious Disease Develop Grant (2012SKLID103).

\section{References}

[1] H. Shang, J. Xu, X. Han, J. Spero Li, K. C. Arledge, and L. Zhang, "HIV prevention: bring safe sex to China," Nature, vol. 485, no. 7400, pp. 576-577, 2012.

[2] Ministry of Health of the People's Republic of China, UNAIDS, and WHO, "National report for HIV/AIDS estimation in China, 2011," Tech. Rep., Chinese Center for Disease Control and Prevention, Beijing, China, 2011.

[3] D. Li, S. Li, Y. Liu et al., "HIV incidence among men who have sex with men in Beijing: a prospective cohort study," British Medical Journal Open, vol. 2, no. 6, Article ID e001829, 2012.

[4] Y. Ruan, Y. Jia, X. Zhang et al., "Incidence of HIV-1, syphilis, hepatitis $\mathrm{B}$, and hepatitis $\mathrm{c}$ virus infections and predictors associated with retention in a 12-month follow-up study among men who have sex with men in Beijing, China," Journal of Acquired Immune Deficiency Syndromes, vol. 52, no. 5, pp. 604610, 2009.

[5] D. L. Li, Y. J. Gao, M. R. Yu et al., "Study on the incidence of HIV and associated risk factors through a prospective cohort among men who have sex with men in Beijing, China," Zhonghua Liu Xing Bing Xue Za Zhi, vol. 33, no. 7, pp. 663-666, 2012.

[6] X. Li, H. Lu, X. Ma et al., "HIV/AIDS-related stigmatizing and discriminatory attitudes and recent HIV testing among men who have sex with men in Beijing," AIDS and Behavior, vol. 16, no. 3, pp. 499-507, 2012.

[7] H. M. Li, R. R. Peng, J. Li et al., "HIV incidence among men who have sex with men in China: a meta-analysis of published studies," PLoS ONE, vol. 6, no. 8, Article ID e23431, 2011.

[8] D. Li, Y. Jia, Y. Ruan et al., "Correlates of incident infections for HIV, syphilis, and hepatitis B virus in a cohort of men who have sex with men in Beijing," AIDS Patient Care and STDs, vol. 24, no. 9, pp. 595-602, 2010.

[9] T. P. Dyer, R. Regan, L. Wilton et al., "Differences in substance use, psychosocial characteristics and HIV-related sexual risk behavior between black men who have sex with men only 
(BMSMO) and black men who have sex with men and women (BMSMW) in six US cities," Journal of Urban Health, vol. 90, no. 6, pp. 1181-1193, 2013.

[10] Q. Li, X. Li, and B. Stanton, "Alcohol use and sexual risk behaviors and outcomes in China: a literature review," AIDS and Behavior, vol. 14, no. 6, pp. 1227-1236, 2010.

[11] S. C. Kalichman, L. C. Simbayi, M. Kaufman, D. Cain, and S. Jooste, "Alcohol use and sexual risks for HIV/AIDS in sub-saharan Africa: systematic review of empirical findings," Prevention Science, vol. 8, no. 2, pp. 141-151, 2007.

[12] A. R. Markos, "Alcohol and sexual behaviour," International Journal of STD and AIDS, vol. 16, no. 2, pp. 123-127, 2005.

[13] R. Jones-Webb, D. Smolenski, S. Brady, M. Wilkerson, and B. R. Rosser, "Drinking settings, alcohol consumption, and sexual risk behavior among gay men," Addictive Behaviors, vol. 38, no. 3, pp. 1824-1830, 2013.

[14] R. G. Deiss, J. L. Clark, K. A. Konda et al., "Problem drinking is associated with increased prevalence of sexual risk behaviors among men who have sex with men (MSM) in Lima, Peru," Drug and Alcohol Dependence, vol. 132, no. 1-2, pp. 134-139, 2013.

[15] P. Vagenas, K. T. Ludford, P. Gonzales et al., "Being unaware of being HIV-infected is associated with alcohol use disorders and high-risk sexual behaviors among men who have sex with men in Peru," AIDS and Behavior, vol. 18, no. 1, pp. 120-127, 2014.

[16] B. Wu, Z. F. Mao, I. R. H. Rockett, and Y. Yue, "Socioeconomic status and alcohol use among urban and rural residents in China," Substance Use and Misuse, vol. 43, no. 7, pp. 952-966, 2008.

[17] Y. Li, Y. Jiang, M. Zhang, P. Yin, F. Wu, and W. Zhao, "Drinking behaviour among men and women in China: the 2007 China chronic disease and risk factor surveillance," Addiction, vol. 106, no. 11, pp. 1946-1956, 2011.

[18] H. Lu, Y. Han, X. He et al., "Alcohol use and HIV risk taking among Chinese MSM in Beijing," Drug and Alcohol Dependence, vol. 133, no. 2, pp. 317-323, 2013.

[19] Y. Ruan, F. Luo, Y. Jia et al., "Risk factors for syphilis and prevalence of HIV, hepatitis B and C among men who have sex with men in Beijing, China: implications for HIV prevention," AIDS and Behavior, vol. 13, no. 4, pp. 663-670, 2009.

[20] E. J. Nehl, F. Y. Wong, N. He, Z. J. Huang, and T. Zheng, "Prevalence and correlates of alcohol use among a sample of general MSM and money boys in Shanghai, China," AIDS Care, vol. 24, no. 3, pp. 324-330, 2012.

[21] C. J. Berg, E. J. Nehl, F. Y. Wong et al., "Prevalence and correlates of tobacco use among a sample of MSM in Shanghai, China," Nicotine and Tobacco Research, vol. 13, no. 1, pp. 22-28, 2011.

[22] H. Y. Tsui and J. T. Lau, "Comparison of risk behaviors and socio-cultural profile of men who have sex with men survey respondents recruited via venues and the internet," BMC Public Health, vol. 10, article 232, 2010.

[23] J. Zhao, L. Chen, W. D. Cai et al., "HIV infection and sexual behaviors among non-commercial men who have sex with men at different venues," Archives of Sexual Behavior, 2013.

[24] J. Jiang, N. Cao, J. Zhang et al., "High prevalence of sexually transmitted diseases among men who have sex with men in Jiangsu Province, China," Sexually Transmitted Diseases, vol. 33, no. 2, pp. 118-123, 2006.

[25] J. Tao, Y. Ruan, L. Yin et al., "Sex with women among men who have sex with men in China: prevalence and sexual practices," AIDS Patient Care and STDs, vol. 27, no. 9, pp. 524-528, 2013.
[26] Y. Liu, X. Li, L. Zhang, S. Li, S. Jiang, and B. Stanton, “Correlates of consistent condom use among young migrant men who have sex with men (MSM) in Beijing, China," The European Journal of Contraception and Reproductive Health Care, vol. 17, no. 3, pp. 219-228, 2012.

[27] J. D. Zheng, L. Pang, J. Xu, K. M. Rou, D. Xiao, and Z. Y. Wu, "Study on the prevalence of HIV and AIDS-related risky sexual behaviors among male university students who have sex with men in Beijing, China," Zhonghua Liu Xing Bing Xue Za Zhi, vol. 32, no. 4, pp. 337-340, 2011.

[28] J. F. Feng, H. J. Lin, Y. F. Zhang, D. H. Qiu, and Q. H. Wu, "Investigation on the related knowledge, behavior and infection of HIV/Syphilis among men who have sex with men in Taizhou city," Shanghai Journal of Preventive Medicine, vol. 20, no. 11, pp. 531-533, 2008.

[29] H. Q. Chen, Y. Li, B. C. Zhang, and X. F. Li, "Study on highrisk behaviour and suicide associated risk factors related to HIV/AIDS among gay or bisexual men," Zhonghua Liu Xing Bing Xue Za Zhi, vol. 32, no. 10, pp. 983-986, 2011.

[30] D. L. Li, X. X. Li, Y. H. Ruan et al., "Factors associated with characteristics of commerical sex encounters among men who have sex with men," Chinese Journal of Natural Medicines, vol. 8, no. 3, pp. 165-168, 2006.

[31] A. H. Lin, P. Yin, W. D. Cai, Z. H. Liang, and T. J. Feng, "Survey of risk factors of HIV infection in MSM in Shenzhen City," China Tropical Medicine, vol. 7, no. 1, pp. 168-169, 2007.

[32] H. He, M. Wang, H. B. Zhang et al., “The unprotected sexual behaviors and its influencing factors among HIV-infected men who have sex with men in Shanghai, China," Zhonghua Yu Fang Yi Xue Za Zhi, vol. 46, no. 11, pp. 976-981, 2012.

[33] X. Y. Sun, X. J. Zhang, M. Li et al., "HIV-related behavior and STD infection status among MSM in Beijing," Occupation and Health, vol. 28, no. 5, pp. 574-577, 2012.

[34] Z. L. Zeng, H. Y. Liang, Y. Yang et al., "Survey of factors associated with unprotected sexual behaviors among men who have sex with men in Beijing," Chinese Journal of Natural Medicines, vol. 10, no. 4, pp. 241-245, 2008.

[35] Z. Q. Dong, M. H. Jin, Z. H. Qiu et al., "Survey of AIDS related knowledge awareness and high-risk behavior in 410 men who have sex with men in Huzhou, Zhejiang," Diease Surveillance, vol. 27, no. 8, pp. 623-626, 2012.

[36] Y. M. Cai, H. Liu, P. Pan et al., "High risk behaviors among 458 men who have sex with men in Shenzhen," South China Journal of Preventive Medicine, vol. 34, no. 2, pp. 13-15, 2008.

[37] Y. Hu, H. Z. Qian, J. Sun et al., "Anal human papillomavirus infection among HIV-infected and uninfected men who have sex with men in Beijing, China," Journal of Acquired Immune Deficiency Syndromes, vol. 64, no. 1, pp. 103-114, 2013.

[38] R. DerSimonian and N. Laird, "Meta-analysis in clinical trials," Controlled Clinical Trials, vol. 7, no. 3, pp. 177-188, 1986.

[39] M. Lipsey and D. Wilson, Practical Meta-Analysis, Sage, Thousand Oaks, Calif, USA, 2001.

[40] R. L. Cook and D. B. Clark, "Is there an association between alcohol consumption and sexually transmitted diseases? A systematic review," Sexually Transmitted Diseases, vol. 32, no. 3, pp. 156-164, 2005.

[41] S. E. Woolf-King and S. A. Maisto, "Alcohol use and highrisk sexual behavior in sub-saharan Africa: a narrative review," Archives of Sexual Behavior, vol. 40, no. 1, pp. 17-42, 2011.

[42] W. Tang, X. Huan, T. Mahapatra et al., "Factors associated with unprotected anal intercourse among men who have sex 
with men: results from a respondent driven sampling survey in Nanjing, China, 2008," AIDS and Behavior, vol. 17, no. 4, pp. 1415-1422, 2013.

[43] Y. Li, J. Xu, K. H. Reilly et al., "Prevalence of HIV and syphilis infection among high school and college student MSM in China: a systematic review and meta-analysis," PloS ONE, vol. 8, no. 7, Article ID e69137, 2013.

[44] Y. Zhang, Z. Dou, K. Sun et al., "Association between missed early visits and mortality among patients of china national free antiretroviral treatment cohort," Journal of Acquired Immune Deficiency Syndromes, vol. 60, no. 1, pp. 59-67, 2012.

[45] F. Yu, E. J. Nehl, T. Zheng et al., "A syndemic including cigarette smoking and sexual risk behaviors among a sample of MSM in Shanghai, China," Drug and Alcohol Dependence, vol. 132, no. 1-2, pp. 265-270, 2013.

[46] S. C. Kalichman, L. C. Simbayi, S. Jooste, and D. Cain, "Frequency, quantity, and contextual use of alcohol among sexually transmitted infection clinic patients in Cape Town, South Africa," American Journal of Drug and Alcohol Abuse, vol. 33, no. 5, pp. 687-698, 2007.

[47] B. Wang, X. Li, B. Stanton, L. Zhang, and X. Fang, "Alcohol use, unprotected sex, and sexually transmitted infections among female sex workers in China," Sexually Transmitted Diseases, vol. 37, no. 10, pp. 629-636, 2010.

[48] X. Luo, S. Duan, Q. Duan et al., "Alcohol use and subsequent sex among HIV-infected patients in an ethnic minority area of Yunnan Province, China," PLoS ONE, vol. 8, no. 4, Article ID e61660, 2013.

[49] L. Vincic, S. Weston, and R. H. Riddell, "Bone core biopsies. Plastic or paraffin?" The American Journal of Surgical Pathology, vol. 13, no. 4, pp. 329-334, 1989.

[50] M. J. Mimiaga, S. L. Reisner, S. Bland et al., "'It's a quick way to get what you want': a formative exploration of HIV risk among Urban Massachusetts men who have sex with men who attend sex parties," AIDS Patient Care and STDs, vol. 24, no. 10, pp. 659-674, 2010.

[51] W. D. Johnson, R. M. Diaz, W. D. Flanders et al., "Behavioral interventions to reduce risk for sexual transmission of HIV among men who have sex with men," Cochrane Database of Systematic Reviews, no. 3, Article ID CD001230, 2008.

[52] R. M. Gerritsen, “The discharge letter," Nederlands Tijdschrift voor Geneeskunde, vol. 133, no. 6, article 314, 1989.

[53] R. D. Stall, J. P. Paul, D. C. Barrett, G. M. Crosby, and E. Bein, "An outcome evaluation to measure changes in sexual risk-taking among gay men undergoing substance use disorder treatment," Journal of Studies on Alcohol, vol. 60, no. 6, pp. 837-845, 1999.

[54] R. M. Young, J. P. Connor, L. A. Ricciardelli, and J. B. Saunders, "The role of alcohol expectancy and drinking refusal selfefficacy beliefs in university student drinking," Alcohol and Alcoholism, vol. 41, no. 1, pp. 70-75, 2006.

[55] M. R. Frone, M. Russell, and M. L. Cooper, "Individual differences in differentiation among alcohol expectancy domains," Addiction, vol. 88, no. 8, pp. 1119-1126, 1993.

[56] C. M. Ryan, J. Huggins, and R. Beatty, "Substance use disorders and the risk of HIV infection in gay men," Journal of Studies on Alcohol, vol. 60, no. 1, pp. 70-77, 1999.

[57] J. A. Boscarino, A. L. Avins, W. J. Woods, C. P. Lindan, E. S. Hudes, and W. Clark, "Alcohol-related risk factors associated with HIV infection among patients entering alcoholism treatment: implications for prevention," Journal of Studies on Alcohol, vol. 56, no. 6, pp. 642-653, 1995.
[58] A. B. Mullens, R. M. Young, M. P. Dunne, and G. Norton, "The drinking expectancy questionnaire for men who have sex with men (DEQ-MSM): a measure of substance-related beliefs," Drug and Alcohol Review, vol. 30, no. 4, pp. 372-380, 2011. 


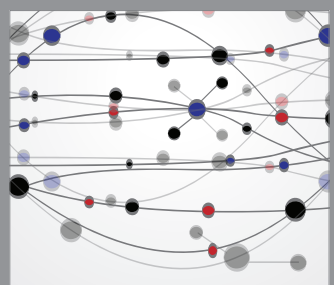

The Scientific World Journal
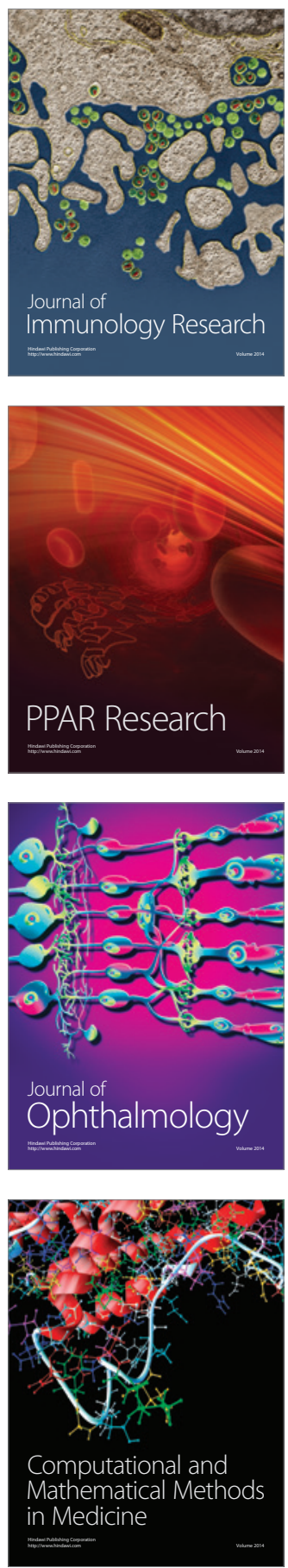

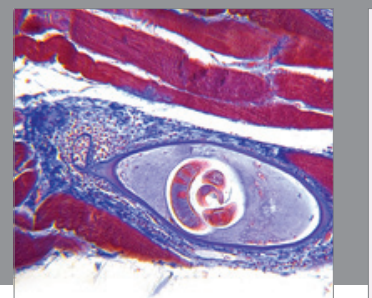

Gastroenterology

Research and Practice
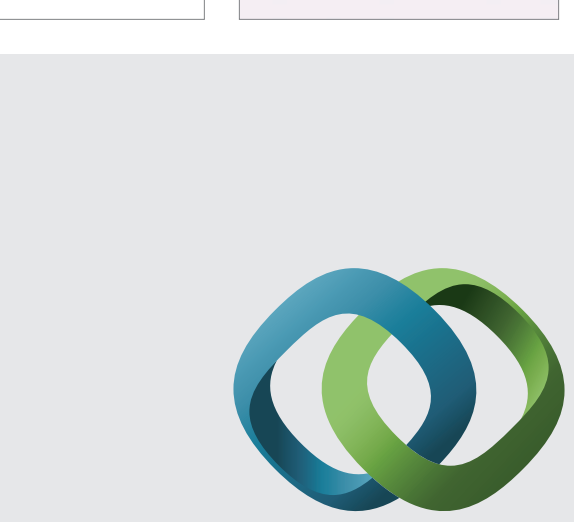

\section{Hindawi}

Submit your manuscripts at

http://www.hindawi.com
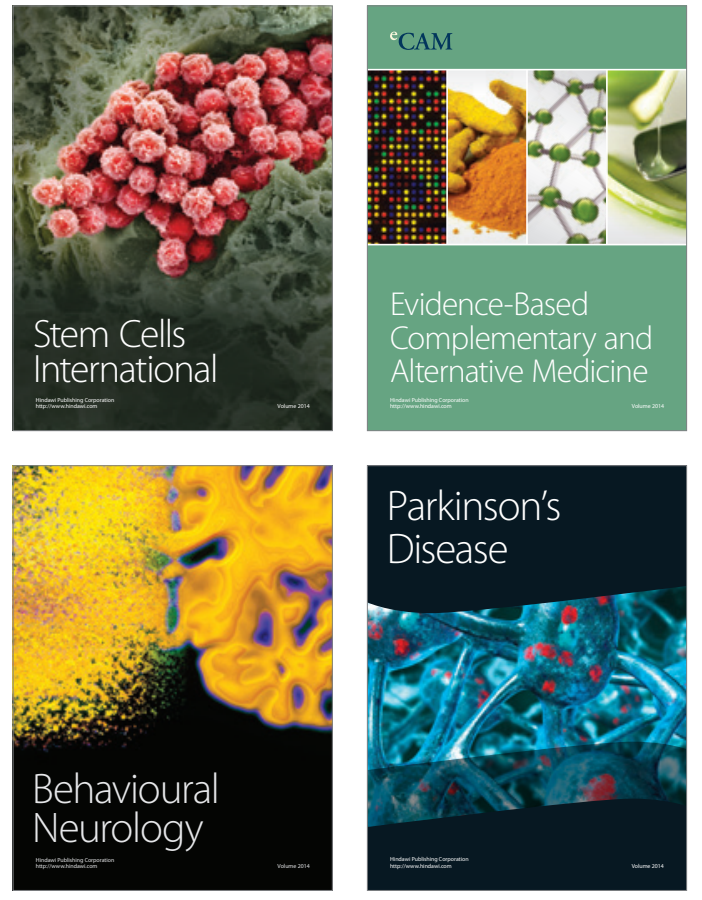
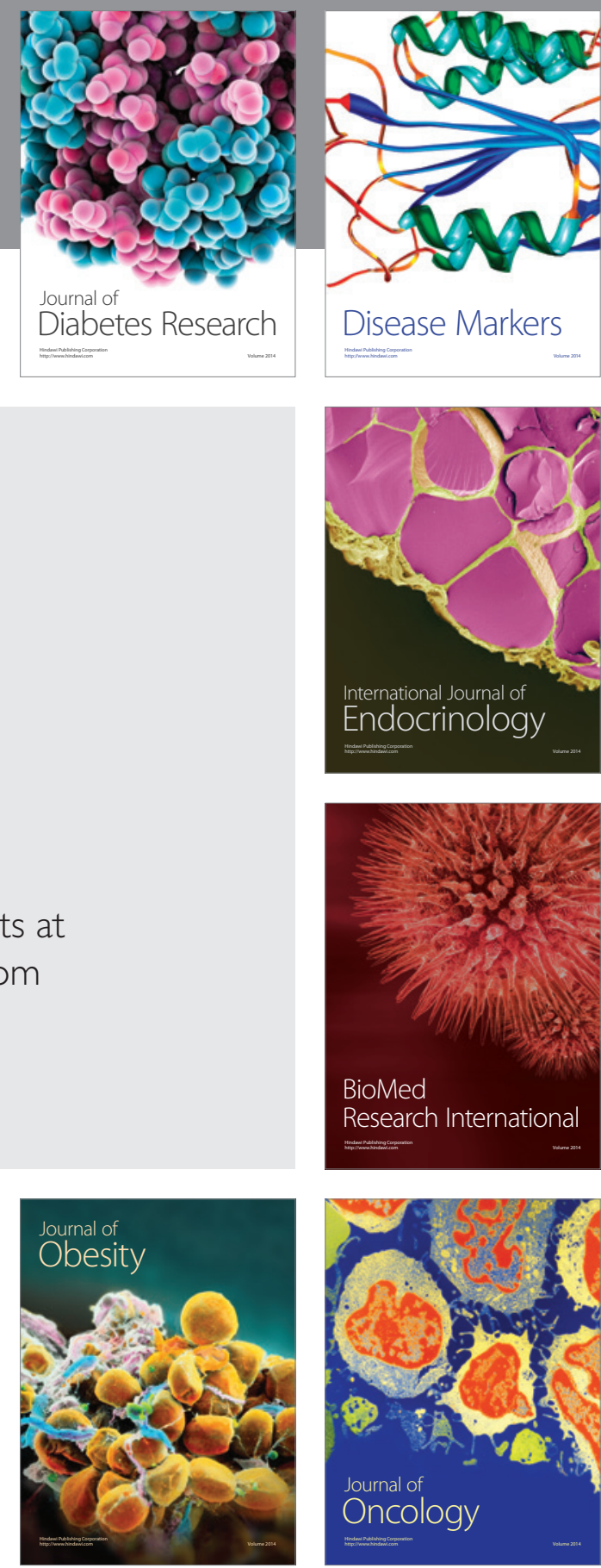

Disease Markers
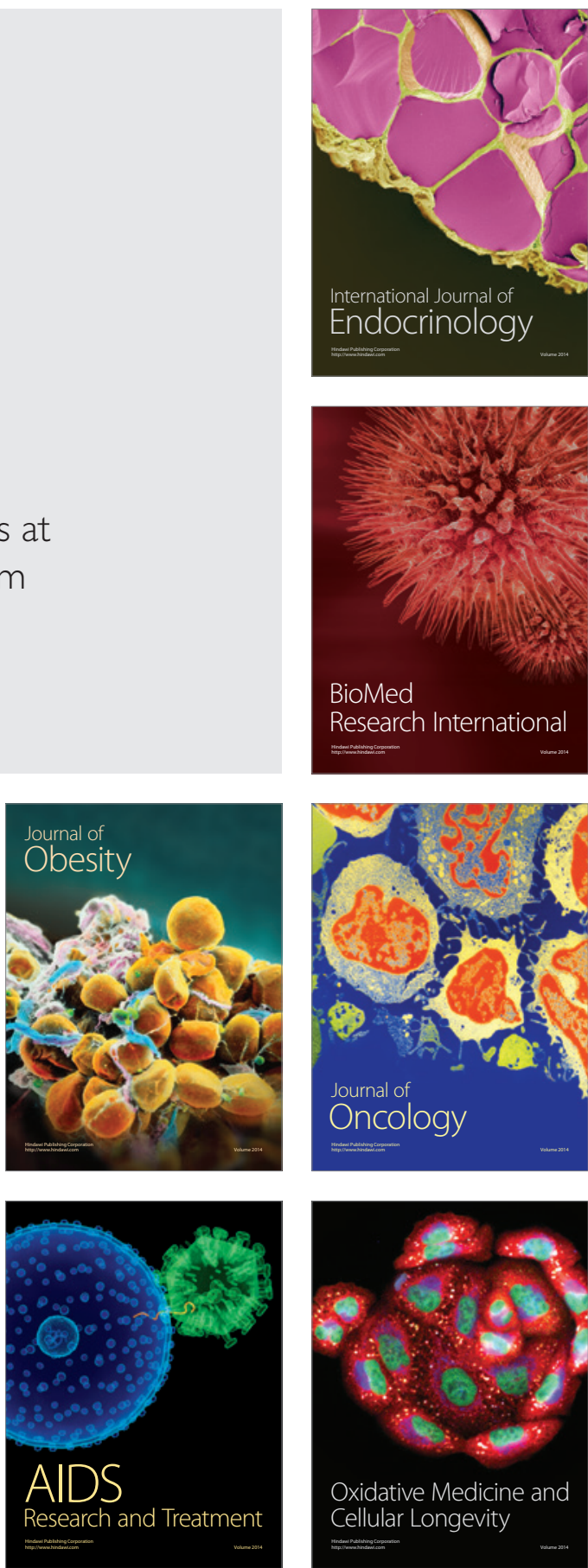